\title{
Anesthesia Outside the Operating Room
}

Editors

MARK S. WEISS

WENDY L. GROSS

\section{ANESTHESIOLOGY CLINICS}

www.anesthesiology.theclinics.com

Consulting Editor

LEE A. FLEISHER

December 2017 • Volume 35 • Number 4 